\title{
Programa de calidad para cumplimiento de NI-43101 en exploración de proyecto de carbón antracita
}

\author{
Quality Program for NI-43101 compliance in exploration activities for \\ anthracite coal project
}

\author{
Marco David Carrasco Rodríguez¹, Jaime César Mayorga Rojas²
}

Recibido: 29/10/2020 - Aprobado: 01/08/2021 - Publicado: 23/12/2021

\begin{abstract}
RESUMEN
Los Programas de Calidad (PDC) son indispensables para el éxito de los objetivos de los proyectos mineros. Estos se componen del plan de gestión de la calidad, gestión y aseguramiento de la calidad (QA) y control de la calidad (QC). Son una herramienta implementada por las empresas mineras con el fin de contar con información valida que permita elaborar los estudios de estimación de recursos y estimación de reservas. La Norma Internacional NI 43101 contiene los lineamientos para la elaboración de los reportes destinados a bolsas de valores, documentos necesarios para el estudio de factibilidad bancaria y para generar valor en las propiedades de las empresas mineras. El contenido de la norma se aplica a la elaboración del Programa de Calidad y así pueda ser implementado durante las actividades de un proyecto minero tales como geología de campo, campañas de perforación, estudios geotécnicos, hidrogeológicos, hidrológicos, topografía, seguridad, evaluaciones ambientales y estudios de agua. En ese sentido, el Programa de Calidad requiere personal altamente especializado y es parte de las políticas y procedimientos de las empresas mineras.
\end{abstract}

Palabras claves: Plan de gestión de calidad; aseguramiento de calidad; control de calidad; norma NI-43101; estudio de factibilidad bancaria.

\begin{abstract}
Quality program is important for achieving the objectives of mining projects. They consist of the quality management plan, quality management and assurance $(\mathrm{QA})$ and quality control $(\mathrm{QC})$. They are a tool implemented by the mining companies in order to have valid information that allows the elaboration of resource mineral estimation and reserve mineral estimation studies. The International Standard NI 43101 contains the guidelines for the elaboration of the reports to stock exchanges and bankable feasibility study. The content of the standard is applied in the elaboration of the Quality Program and thus it can be implemented during the activities of a project such as field geology, drilling campaigns, geotechnical, hydrogeological, hydrological studies, topography, security, environmental evaluations and water quality studies. In this sense, the Quality Program requires highly specialized staff and is part of the policies and procedures of mining companies.
\end{abstract}

Keywords: Quality management plan; quality assurance; quality control; NI-43101 standard; bankable feasibility study.

\footnotetext{
1 Universidad Nacional Mayor de San Marcos, Facultad de Ingeniería Geológica, Minera, Metalúrgica y Geográfica, Unidad de Posgrado, Lima, Perú. Alumno. Autor para correspondencia: marcodavid.carrasco@unmsm.edu.pe

2 Universidad Nacional Mayor de San Marcos, Facultad de Ingeniería Geológica, Minera, Metalúrgica y Geográfica, EAPIG, Lima, Perú. Docente. E-mail: jaime.mayorga@unmsm.edu.pe - ORCID: https://orcid.org/0000-0001-8423-3343
} 


\section{INTRODUCCIÓN}

Los proyectos mineros consideran diferentes actividades tales como la exploración, explotación, procesamiento, distribución y comercialización de minerales. De manera holística la gestión de un proyecto incluye un Programa de Calidad (PDC). Este programa se aplica a cada una de las etapas del proyecto con el fin planificar, gestionar, asegurar y controlar la calidad, de esta manera contar con información confiable para el desarrollo de informes destinados a los shareholders y stakeholders.

La Organización Internacional de Normalización (ISO), define la gestión de la calidad como las actividades coordinadas para dirigir y controlar la calidad, la cual puede incluir políticas, objetivos y procesos. La ISO indica que el aseguramiento de la calidad (QA) es parte de la gestión de la calidad orientada a dar confianza en el cumplimiento de los requisitos de la calidad y finalmente, el control de la calidad (QC) está orientado al cumplimiento de la calidad (ISO, 2015).

En el proyecto de carbón se implementó un Programa de Calidad para la ejecución de las actividades de exploración. Para tal fin, se organizó el proyecto considerando diversas áreas especializadas incluyendo el área de calidad (AC) cuya función principal fue la implementación del PDC.

El objetivo del proyecto de exploración fue crear valor a la empresa mediante el desarrollo de una base de datos optimizada del depósito minero, que permita evaluar y obtener la factibilidad del proyecto de explotación de carbón antracita. Es importante mencionar que dentro de los informes más importantes en el proyecto de carbón se encuentran la estimación de Recursos, estimación de Reservas y los informes de ingeniería a nivel factibilidad. En la presente publicación se describe las actividades del PDC durante la etapa de exploración del proyecto minero de carbón antracita ubicado en la cuenca de Alto Chicama en La Región La Libertad. Las actividades del PDC permitieron gestionar, asegurar y controlar la información obtenida durante las actividades de mapeo geológico superficial, perforaciones diamantinas, análisis de laboratorio, auditorias, estudios geotécnicos, topográficos para ser utilizadas en la estimación de Recursos Minerales bajo los estándares de la norma internacional NI-43101 (CSA, 2011).

En la presente publicación, se desarrolló el PDC para el proyecto de carbón antracita, considerando la génesis de carbón antracita en el Perú, la cual tuvo solo tres periodos cortos durante los cuales se formaron los carbones (DuninBorkowsk et al., 2007).

1. Misisipano Inferior hace unos $330 \mathrm{Ma}$. (Viseano)

2. Cretáceo de 140 a 130 Ma (del Beriasiano a Valanginiano Inferior)

3. Neógeno (principalmente Mioceno) y Cuaternario durante los últimos $30 \mathrm{Ma}$.

El depósito de Carbón Antracita descrito en el presente artículo corresponde a la edad geológica del Cretáceo temprano y en Valanginiano medio. El proyecto de carbón se ubica en la cuenca del Alto Chicama donde se identificó la litología de las formaciones pertenecientes al Jurásico Superior (formación Chicama) y al grupo Goyllarisquizga, tales como la formación Chimú, formación Santa, formación Carhuaz, formación Farrat. Importante mencionar que la formación Chimú es la que almacena los mantos de carbón en el depósito estudiado (Carrascal Miranda et al., 2000).

Desde el punto de vista tectónico, el área de estudio presenta sobre- escurrimientos y repeticiones de las series productivas con carbón. En los mantos carboníferos se emplazan preferentemente las fallas y los sills ígneos. Diques concordantes discordantes con la estratificación plegada y perturbada. El tectonismo ha deformado los mantos y molido el carbón, mezclándolo con las lutitas encajonantes.

En el entorno de la zona de estudio, se tienen rocas pertenecientes a las formaciones Chicama y Chimú. Se reconoció rocas sedimentarias, metamórficas e ígneas. Las rocas metamórficas son las más predominantes en el área del proyecto. Dentro de las rocas metamórficas se encuentran cuarcitas, carbón antracita (6 mantos) y pizarras.

También, se identificó rocas Ígneas las cuales son una serie de pequeños cuerpos ígneos de composición andesítica-dacítica que han intruído las unidades estratigráficas. Por otro lado, las rocas sedimentarias identificadas fueron cuaternario del tipo coluvial, brecha craquelada, areniscas y lutitas.

\section{MÉTODOS}

El PDC descrito en la presente publicación fue aplicado en las actividades de exploración de geología de campo y campaña de perforación diamantina del proyecto. Las actividades de exploración se desarrollaron en las concesiones ubicadas en la Región La Libertad. Por su parte, las perforaciones diamantinas se llevaron a cabo en una de las concesiones mineras, la cual fue priorizada como zona de estudio.

Sobre las actividades de exploración, la norma NI43101 (CSA, 2011) detalla los aspectos a incluir en los reportes técnicos aceptados internacionalmente. En el reporte se debe incluir la naturaleza, extensión y el trabajo relevante desarrollado con fines de exploración. Los métodos de muestreo y la calidad del muestreo son la base para la estimación de recursos y reservas. Otro aspecto importante es la representatividad de las muestras, según la norma, es necesario detallar los factores que podrían afectar la calidad de los resultados de estas.

Asimismo, considerando que los proyectos mineros inician con la aprobación del "Project Chárter" o "Documento de Inicio del Proyecto" mediante el cual se autoriza a la gerencia del proyecto a realizar las actividades programadas. Para las actividades de exploración de geología de campo y en la campaña de perforación se consideró las etapas descritas en la gestión de proyectos mencionada en la guía de gestión de proyectos de PMI (PMI, 2017) los cuales se muestran en la Figura 1. 


\subsection{Programa de calidad}

Basado en el estándar de PMI Capitulo 8 de PMBOK, se diseñó el PDC del proyecto. El PDC consideró el plan de gestión de la calidad (PGC), gestión de calidad / aseguramiento de la calidad (QA) y control de la calidad (QC). El Plan de Gestión de la Calidad (PGC) buscó identificar los estándares de calidad para el proyecto, el objetivo del aseguramiento de la Calidad (QA) fue que los resultados y actividades se realicen siguiendo las mejores prácticas en la industria y satisfagan los estándares de calidad, este incluye actividades de auditoria, y el Control de la Calidad (QC) buscó verificar que las actividades de calidad sean eficaces, asimismo, monitorear y controlar los resultados obtenidos.

El alcance del PDC desarrollado durante la etapa de exploración del proyecto se muestran en la Figura 2.

Por otro lado, se incluyó dentro del PDC las siguientes actividades: Integración del Proyecto, geología de campo, perforaciones diamantinas, administración de base de datos, interpretación geológica y estimación de Recursos.

En la Tabla 1 se muestra la relación entre los componentes del PDC y las actividades de exploración del proyecto de carbón antracita.
El PGC incluyó los protocolos desde las actividades previas al inicio de la perforación hasta la validación de los resultados de análisis de laboratorio que fueron utilizados en la estimación de los recursos y reservas. Las actividades de geología de campo y perforación diamantina, en las cuales se aplicó el PGC fueron: selección de empresas contratistas, elaboración de protocolos, actividades de perforación, supervisión, estudios topográficos, transporte de cores, logueo geológico, logueo geotécnico, actividades en coreshack, identificación de muestras, corte o homogenización, preparación de muestras, análisis de laboratorio, cadena de custodia, base de datos, modelo geológico y estimación de recursos.

\subsection{Actividades previas a la exploración}

La definición del alcance de las actividades de exploración permitió definir de manera adecuada el PDC. Se integró las diversas áreas del proyecto tales como operaciones, control de calidad, finanzas, legal, logística, ambiental y social. El PDC se aplicó desde el inicio del proyecto y para esto se elaboró una lista de chequeo de los aspectos asociados a temas de calidad y hacerlo de manera temprana permitió tener una gestión óptima. En ese sentido, se elaboró protocolos para definir las variables a incluir dentro de la gestión, aseguramiento y control de la calidad. Los protocolos son documentos que permitieron conocer las

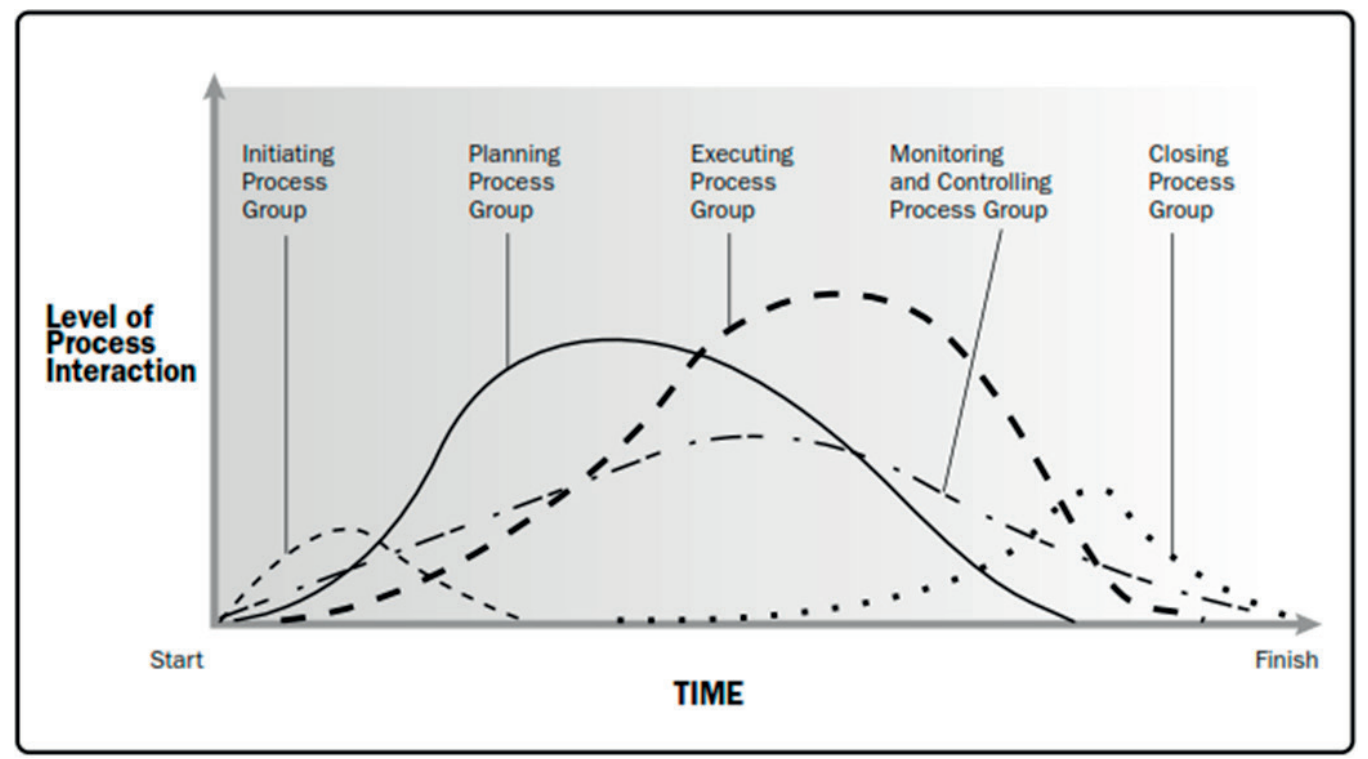

Figura 1. Etapas de los proyectos (PMI, 2017)

\begin{tabular}{|c|c|c|}
\hline \multicolumn{2}{|c|}{ Programa de Calidad } \\
\hline \begin{tabular}{c|c|} 
Plan de Gestión de la Calidad \\
(PGC)
\end{tabular} & $\begin{array}{c}\text { Gestión y Aseguramiento } \\
\text { de la Calidad (QA) }\end{array}$ & $\begin{array}{c}\text { Control de la Calidad } \\
\text { (QC) }\end{array}$ \\
\hline Organigrama & $\begin{array}{c}\text { Aseguramiento de la } \\
\text { Calidad (QA) } \\
\text { Reportes } \\
\text { Estándares } \\
\text { Métricas }\end{array}$ & $\begin{array}{c}\text { Control de la Calidad (QC) } \\
\text { Análisis de Datos } \\
\text { Reporte de la Calidad } \\
\text { Solicitudes de Cambio }\end{array}$ \\
\hline
\end{tabular}

Figura 2. Organización del programa de calidad (PDC) 
Tabla 1. Actividades de exploración y los componentes del programa de calidad

\begin{tabular}{|c|c|c|c|}
\hline $\begin{array}{c}\text { Actividades } \\
\text { de Exploración }\end{array}$ & $\begin{array}{l}\text { Plan de Gestión de la Calidad } \\
\text { (PGC) }\end{array}$ & $\begin{array}{l}\text { Gestión y Aseguramiento de la Calidad } \\
\text { (QA) }\end{array}$ & $\begin{array}{l}\text { Control de la Calidad } \\
\text { (QC) }\end{array}$ \\
\hline $\begin{array}{l}\text { 1. Integración del } \\
\text { proyecto }\end{array}$ & $\begin{array}{l}\text { 1.1.Elaboración de estándares. } \\
\text { 1.2.Preparación de protocolos de muestreo. } \\
\text { 1.3.Sistema Topográfico. } \\
\text { 1.4.Preparación de protocolos de logueo } \\
\text { geológico y geotécnico. } \\
\text { 1.5. Cadena de custodia. } \\
\text { 1.6. Envío de muestras a laboratorio. } \\
\text { 1.7.Protocolos de registro de datos. } \\
\text { 1.8.Contratos de servicios con terceros. }\end{array}$ & & \\
\hline 2.Geología de campo & & $\begin{array}{l}\text { 2.1. Toma de muestras. } \\
\text { 2.2. Ubicación de coordenadas de puntos de } \\
\text { muestreo. } \\
\text { 2.3. Logueo de muestras. } \\
\text { 2.4.Preparación de muestras, análisis y } \\
\text { seguridad. } \\
\text { 2.5. Envío de muestras al laboratorio. } \\
\text { 2.6. Cadena de custodia. } \\
\text { 2.7. Administración del coreshack. }\end{array}$ & $\begin{array}{l}\text { 2.8. Análisis estadístico de resultados. } \\
\text { 2.9. Envío de blancos. Envío de duplicados. } \\
\text { 2.10.Control de desviaciones sobre los } \\
\text { estándares. } \\
\text { 2.11.Verificación de repetibilidad de las } \\
\text { muestras enviadas a laboratorio. }\end{array}$ \\
\hline $\begin{array}{l}\text { 3.Perforaciones } \\
\text { Diamantina }\end{array}$ & & $\begin{array}{l}\text { 3.1. Supervisión de la perforación. } \\
\text { 3.2. Ubicación de coordenadas de } \\
\text { plataformas y collar. } \\
\text { 3.3. Logueo geológico y geotécnico de los } \\
\text { cores. } \\
\text { 3.4.Preparación de muestras, análisis y } \\
\text { seguridad. } \\
\text { 3.5. Envío de muestras a laboratorio. } \\
\text { 3.6.Administración del coreshack. }\end{array}$ & $\begin{array}{l}\text { 3.7. Análisis estadístico de resultados. } \\
\text { 3.8. Envío de blancos. } \\
\text { 3.9. Envío de duplicados. } \\
\text { 3.10. Control de desviaciones sobre los } \\
\text { estándares. } \\
\text { 3.11.Verificación de repetibilidad de las } \\
\text { muestras enviadas a laboratorio. }\end{array}$ \\
\hline $\begin{array}{l}\text { 4.Administración de la } \\
\text { Base de Datos }\end{array}$ & & $\begin{array}{l}\text { 4.1. Registro de muestras en base de datos } \\
\text { 4.2. Cadena de custodia de las muestras. }\end{array}$ & $\begin{array}{l}\text { 4.3. Elaboración de tablas estadísticas y } \\
\text { cuadros de resultados. }\end{array}$ \\
\hline $\begin{array}{l}\text { 5.Interpretación } \\
\text { Geológica }\end{array}$ & 5.1 Estándares de modelamiento geológico & $\begin{array}{l}\text { 5.2. Elaboración del modelo geológico. } \\
\text { 5.3.Aplicación de los estándares. } \\
\text { 5.4.Auditorías externas. }\end{array}$ & $\begin{array}{l}\text { 5.5. Uso de estándares. } \\
\text { 5.6. Verificación de los resultados de campo } \\
\text { y los mostrados en el modelo geológico. }\end{array}$ \\
\hline $\begin{array}{l}\text { 6. Estimación de } \\
\text { Recursos }\end{array}$ & 6.1 Estándares de estimación de Recursos & $\begin{array}{l}\text { 6.2. Elaboración de estimación de Recursos. } \\
\text { 6.3. Auditoria de los criterios para la } \\
\text { estimación de los Recursos. }\end{array}$ & $\begin{array}{l}\text { 6.4. Uso de Normas Internacionales. } \\
\text { 6.5. Verificación de los resultados del } \\
\text { modelo geológico y los mostrados en la } \\
\text { estimación de Recursos. }\end{array}$ \\
\hline
\end{tabular}

actividades en temas de control documentario, aspectos técnicos, logística, cadena de custodia, manejo de base de datos, aspectos de salud, seguridad y medio ambiente que fueron propias de cada actividad durante la exploración.

La lista de chequeo incluyó los criterios de evaluación típicos de los proyectos mineros y los cuales se encuentran en las mejores prácticas de la industria (CIM - Canadian Institute of Mining, 2018) dentro de los cuales se encuentran: A. Aspectos Generales: descripción del proyecto, localización, accesibilidad, clima, visitas B. Datos del Proyecto: geología, topografía, muestreo que incluye métodos, preparación, análisis, verificación de muestras, densidad, seguridad de muestras, administración de base de datos, C. Interpretación: interpretación y modelo geológico, modelo de Recursos, D: Clasificación de Recursos y Reservas, oportunidades y riesgos E. Extracción : mina, procesamiento, recuperación $\mathrm{F}$. infraestructura y administración G. Aspectos Ambientales, H. Aspectos Sociales, I Viabilidad Económica J. Riesgos K Consideraciones y recomendaciones.

\subsection{Actividades de exploración}

Las actividades iniciales en el proyecto fueron: geología de campo cuyo objetivo principal fue conocer con mayor profundidad el depósito. Se desarrolló geología de campo en 11 concesiones mineras con un área de 2,500 hectáreas.
Se identificó las zonas potenciales mediante interpretación geológica, análisis de laboratorio, elaboración de modelo geológico y secciones geológicas que permitieron entender el depósito. Con esta información se elaboraron los documentos para desarrollar la campaña de exploración mediante perforaciones diamantina.

Los criterios utilizados para definir las zonas potenciales fueron los siguientes: mayor potencial geológico (cantidad y calidad), menor cantidad perforaciones, menor riesgo social, menor riesgo ambiental y de seguridad, ubicación de las futuras instalaciones mineras del proyecto minero, accesibilidad, servicios auxiliares, permisos y planificación de futuras campañas de exploración.

En adición a la campaña de perforación diamantina, se planificó estudios geotécnicos, pruebas hidrogeológicas, estudios hidrogeológicos, instalaciones de piezómetros, análisis de laboratorio, auditoria de cumplimiento de NI43101 y revisión del modelo geológico y estimación de Recursos.

\subsection{Supervisión}

El PDC fue aplicado durante la etapa de geología de campo y la ejecución de la campaña de perforación, esta última fue desarrollada por una empresa especializada. Personal de la empresa supervisó las actividades de perforación mediante 
quickLog (logueo rápido), que tuvo por objetivo verificar la litología obtenida de cada perforación y que fue estimada de manera previa a la perforación. En esta actividad se contó con formatos de registro y de control. Los geólogos de supervisión verificaron aspectos técnicos de perforación y los controles tales como la ubicación del sondaje, azimuth, DIP, profundidad de cada muestra obtenida, recuperación de la muestra, resultado entre la longitud y el espesor verdadero de la mineralización, consumo de lodos, agua, aditivos, rotación y velocidad de perforación con el fin de obtener los cores de manera adecuada.

La supervisión incluyó la preparación de collares, supervisión y preparación de plataformas, quicklog, transporte de cores, y cadena de custodia, cuyo objetivo fue asegurar la calidad de los cores desde la plataforma de perforación hasta la entrega de estos al coreshack del proyecto.

La gestión de la calidad y el aseguramiento de la calidad consideró que el core obtenido en la perforación sea colocado de manera adecuada y correlativamente en las cajas de core.

Finalmente, las cajas de cores fueron transportadas desde la plataforma de perforación hasta el coreshack, esta actividad se realizó con la adecuada cadena de custodia. El protocolo de transporte de muestras dentro del PGC incluyó las condiciones de transporte con el fin de conservar la integridad de los cores y evitar algún daño que impacte sobre la calidad de los resultados.

\subsection{Topografía}

Para el desarrollo de una topografía adecuada y siguiendo las mejores prácticas de la industria se instaló puntos georreferenciados certificados y obtenidos del Instituto Geográfico Nacional (IGN). La actividad de topografía permitió tener información de los collares, y tener certeza en los parámetros base para el desarrollo de la estimación de recursos minerales.

2.6. Preparación de muestras, análisis y seguridad Las muestras recibidas de las actividades de geología de campo y de la campaña de perforación, fueron codificadas y almacenadas. Se realizó fotografías de alta resolución de cada muestra, con el fin de tener evidencia del estado original de la muestra antes de manipulación. En la Figura 3 se muestra la fotografía de alta calidad de una caja de cores obtenida durante la perforación.

\subsection{Logueo geológico y logueo geotécnico}

Durante las actividades de geología de campo y durante la campaña de perforación se obtuvieron 242 muestras de carbón, las cuales permitieron obtener información geológica del depósito. Las muestras de campo y los Cores obtenidos en la perforación representan la información más importante para los objetivos de estimación de recursos y reservas del proyecto. El proyecto contó con un coreshack donde se almacenaron todas las muestras de las diferentes etapas del proyecto y que fueron utilizadas en auditorías externas para cumplimiento de norma NI-43101.

Como parte de la gestión y aseguramiento de la calidad (QA), se utilizó el manual de logueo geológico desarrollado durante la etapa de planificación del proyecto. Luego de terminada cada perforación diamantina, se desarrolló el logueo geológico a detalle y así conocer la litología del depósito. El logueo geológico también permitió identificar las muestras representativas, las cuales serían analizadas en el laboratorio.

El logueo geotécnico se realizó con el fin de conocer las características del depósito, este logueo permitió conocer las características tales como el RMR y Q', esta información junto con los estudios hidrogeológicos permitirá posteriormente definir el método de minado y el plan de minado del proyecto.

El Programa de Calidad aplicado al logueo geotécnico consideró la adecuada conservación de las muestras, y así identificar de manera adecuada las fracturas, fallas, aspectos físicos del depósito, con una adecuada conservación del core.

Posterior a la identificación de las muestras obtenidas durante la ejecución de las perforaciones, se continuó con la etapa de preparación de las muestras, en esta etapa se utilizó una base de datos para el registro de la información

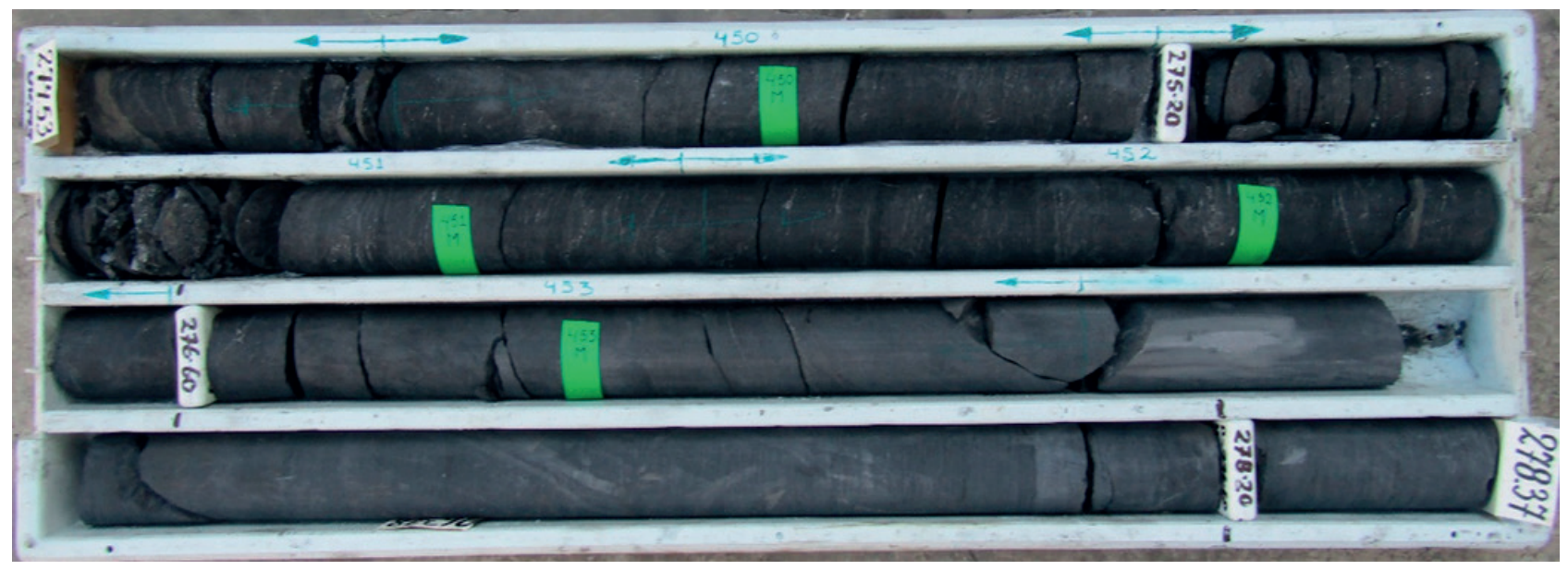

Figura 3. Fotografías de alta calidad de caja de cores. 
geológica en formato digital o físico sobre el tipo de muestra, se determinó la representatividad, calidad y la cantidad de muestra necesaria para los análisis o ensayos a realizar. Se verificó la existencia de elementos de contaminación que pueden afectar el resultado final de análisis de las muestras.

Sobre el método de homogenización o cuarteo, se implementaron procedimientos cuyo objetivo principal fue buscar que la muestra seleccionada pueda ser divida en submuestras, considerando que de cada una de estas se pueda obtener el mismo resultado físico-químico y con un error dentro de los márgenes acéptales en la industria. En la Figura 4 se presenta una muestra de carbón antracita desagregado y homogenizado, el cual fue obtenido durante la etapa de perforación diamantina. El método de homogenización que se utilizó en el proyecto de carbón dependió de las características físicas de la muestra. Las características de las muestras de carbón eran del tipo desagregada o compacta.

En la Figura 5, se presenta una muestra de carbón antracita en estado compacto. Para muestras compactas se utilizó una máquina de corte considerando que la muestra debe ser representativa en las partes que sea dividida. Esta actividad fue supervisada por los geólogos del área de calidad.

Finalmente, se realizaron las actividades de embolsado, etiquetado y transporte de muestras geología de campo y perforación, para lo cual se utilizó los procedimientos que permitieron conservar la calidad de muestra.

\subsection{Análisis de muestras}

Para el análisis de muestras se seleccionó laboratorios especializados en análisis de carbón. Se verificó los protocolos de QA/QC de cada laboratorio y así realizar la evaluación estadística de los resultados. En la Tabla 2 se presentan los controles rutinarios de inserción de estándares, duplicados y blancos como parte del monitoreo y control de la calidad.

Para los análisis de muestras de carbón se utilizaron métodos específicos de acuerdo a las características físico-

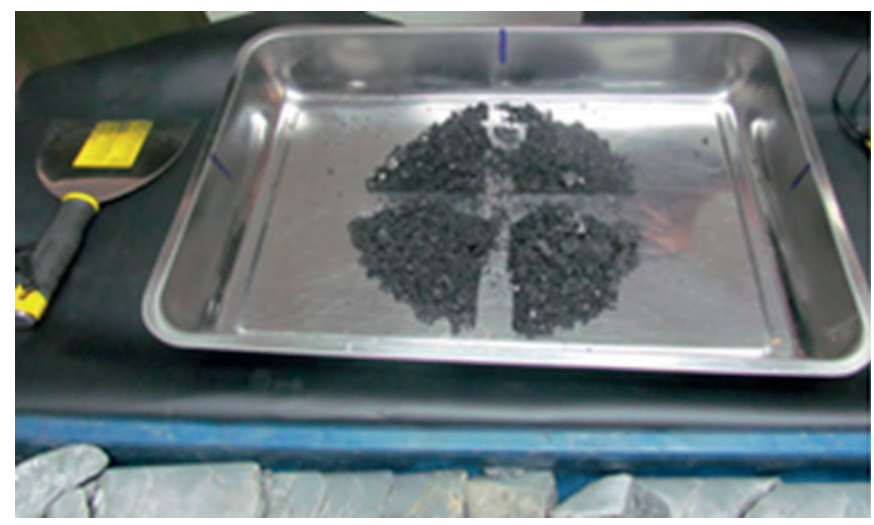

Figura 4. Muestra de carbón desagregada

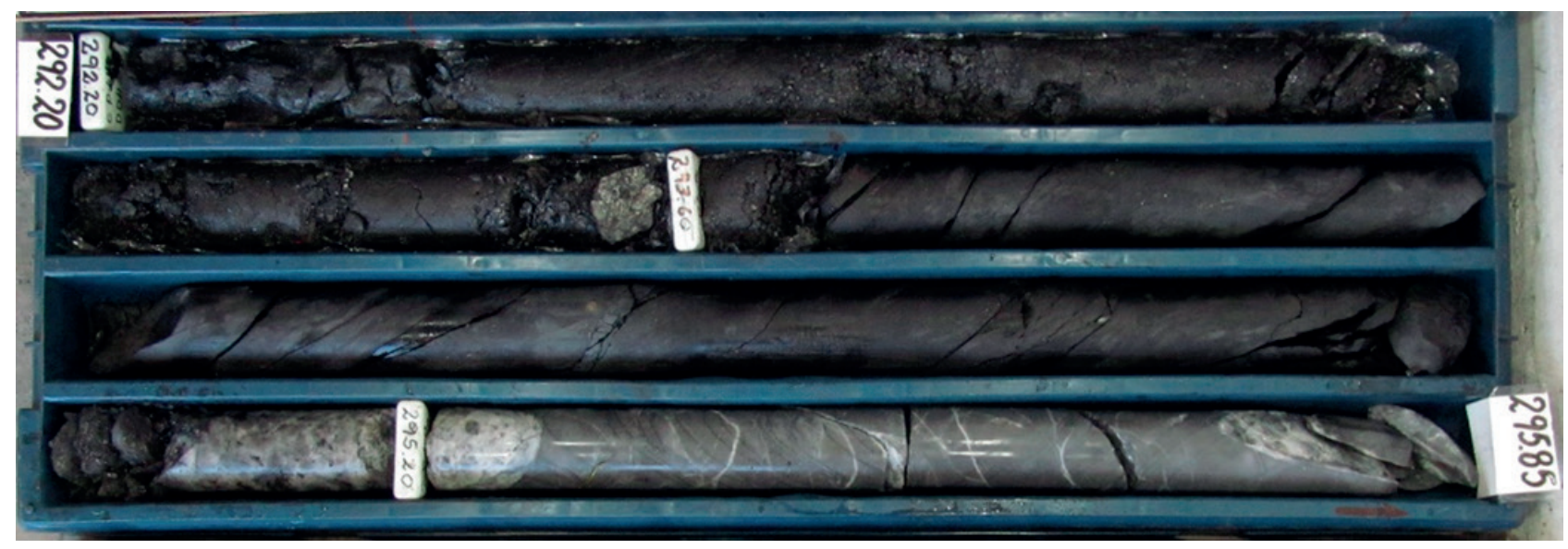

Figura 5. Muestra de carbón antracita compacta

Tabla 2. Controles rutinarios de análisis de muestras

\begin{tabular}{llll}
\hline Procedimiento & Standard & Duplicados & Blancos \\
\hline Laboratorio Empresa & 1 estándar cada 20 muestras & Duplicados al azar y 1 Duplicado / 20 muestras & 2 blancos / 10 Muestras \\
Laboratorio Externo & 1 estándar cada 20 muestras & Duplicados al azar por Batch & 2 blancos / Batch \\
\hline
\end{tabular}


químicas de las muestras. Los métodos para cada tipo de análisis se presentan en la Tabla 3.

Tabla 3. Análisis Físico y método de análisis

\begin{tabular}{cll}
\hline ítem & Análisis Químico & Método Analítico \\
\hline 1 & Humedad, \% & ASTM D 3302 \\
2 & Cenizas, \% & ASTM D 7582-15(A) \\
3 & Azufre, \% & ASTM D 4239 \\
4 & Material Volátil, \% & ASTM D 3175-11(A) \\
5 & Carbón Fijo, \% & ASTM D 3172-07 a (Dif) \\
6 & Poder Calorífico, Kcal/kg & ASTM D 5865 \\
7 & Cloro, \% & ASTM D 4208-13 (A) \\
8 & Preparación de muestras & ASTM D 2013 \\
\hline
\end{tabular}

El programa de calidad incorporó una comparación de los resultados obtenidos entre dos laboratorios externos, de esta manera verificar la precisión y repetitividad de los resultados. Los resultados se presentan en el capítulo resultados.

En la Tabla 4 se presenta el número de muestras obtenidas durante geología de campo y la campaña de perforación. Asimismo, se muestra el número de muestras analizadas y el número de muestras duplicado y blancos.

\subsection{Seguridad}

La cadena de custodia fue uno de los procedimientos más importantes en el proyecto. La cadena de custodia fue supervisada por el área de calidad de la empresa. Se implementó los procedimientos, formatos y la organización para la administración de muestras y su respectiva cadena de custodia. La cadena de custodia comenzó desde la obtención de las muestras en campo o durante la perforación y culminó en la recepción de los análisis de laboratorio.

\subsection{Verificación de datos}

Los reportes NI-43101 incluyen un capítulo sobre verificación de datos, en este capítulo se debe describir las actividades que realizó el Qualified Person (QP) con el fin de verificar la información del proyecto, y que normalmente se encuentra en la base de datos. Se debe describir en este capítulo los procedimientos, las limitaciones o fallas que se cometieron durante la verificación explicando las razones del por qué ocurrieron. Es parte de este ítem, la opinión del QP sobre la adecuada calidad de los datos obtenidos en la ejecución de las actividades. En el caso del proyecto de exploración de carbón antracita, se realizó la verificación de datos de manera interna con el área de control de calidad y, asimismo, se contrató a una empresa auditora internacional para la verificación de los resultados obtenidos, obteniendo la opinión favorable del QP.

Se realizó la verificación de los datos como parte de la gestión y aseguramiento de la calidad (QA), con esta actividad se elaboró la interpretación, modelo geológico y la estimación de Recursos. En esta etapa se verificó la consistencia de la información obtenida en campo, se verificó que no existió ningún tipo de alteración en la base de datos del proyecto.

La auditoría externa incluyó la verificación de datos, esto se realizó mediante la visita de un QP al coreshack del proyecto. Durante la visita, el QP realizó un muestreo aleatorio de los resultados obtenidos durante el logueo geológico, logueo geotécnico, hojas de muestreo, las actividades generales del proyecto y la información que pudiera haber afectado la calidad de la estimación de Recursos.

\section{RESULTADOS}

Es importante mencionar que, durante las actividades de geología de campo o perforación es común tener errores o desviaciones, los más comunes pueden ser pérdidas o mala homogenización de las muestras. Las perdidas dependen del método de muestreo, puede incluir polvo, exagerado uso de agua durante la perforación, mala manipulación de muestras, inadecuado sistema de embolsado de las muestras, mala codificación demuestras, alteraciones en las bases de datos y mal corte de muestreas (Abbott, 2007).

En ese sentido, y como parte del programa de calidad, se comparó los resultados de los dos laboratorios externos seleccionados utilizando la misma metodología de análisis y de esta manera verificó la confiabilidad de los resultados obtenidos. Sobre la comparación de los resultados se aplicó el método denominado diferencia porcentual media o Mean Percent Difference (MPD), el cual nos permitió conocer la variabilidad de un valor referente a otro. Para el cálculo de MPD de los resultados obtenidos de los dos laboratorios externos evaluados resultó $10 \%$. Los valores obtenidos por el laboratorio seleccionado para el análisis de las muestras de proyecto muestran resultados aceptables.

En la Figura 6 se muestra los resultados de los laboratorios externos.A continuación, y como parte del control de calidad (QC) se muestra resultados de las muestras originales y sus duplicados. Se realizó el análisis estadístico para las principales características como son poder calorífico, contenido de cenizas, material volátil y azufre. Las Figuras 7 y Figura 8 muestran los resultados de los análisis de poder calorífico y material volátil respectivamente.

Asimismo, como parte del control de la calidad (QC), para las actividades de geología de campo y para las perforaciones diamantina se utilizaron como herramientas de control: histogramas y scatter plot, ambas herramientas permitieron analizar y dar seguimiento a los resultados durante la ejecución de las actividades de exploración.

Tabla 4. Muestras, Duplicados, Blancos obtenidos durante las actividades de exploración

\begin{tabular}{lccc}
\hline Actividad & Muestras Analizadas en laboratorio & Duplicados & Blancos \\
\hline Geología de campo & 162 & 13 & 26 \\
Campaña de Perforación Diamantina & 80 & 9 & 9 \\
\hline
\end{tabular}




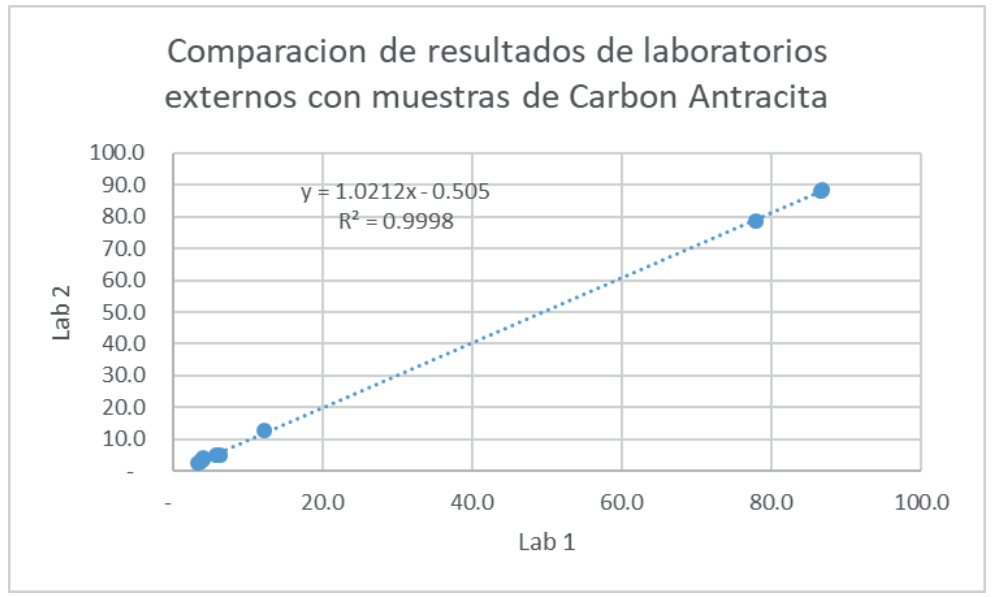

Figura 6. Comparación de resultados de laboratorios externos

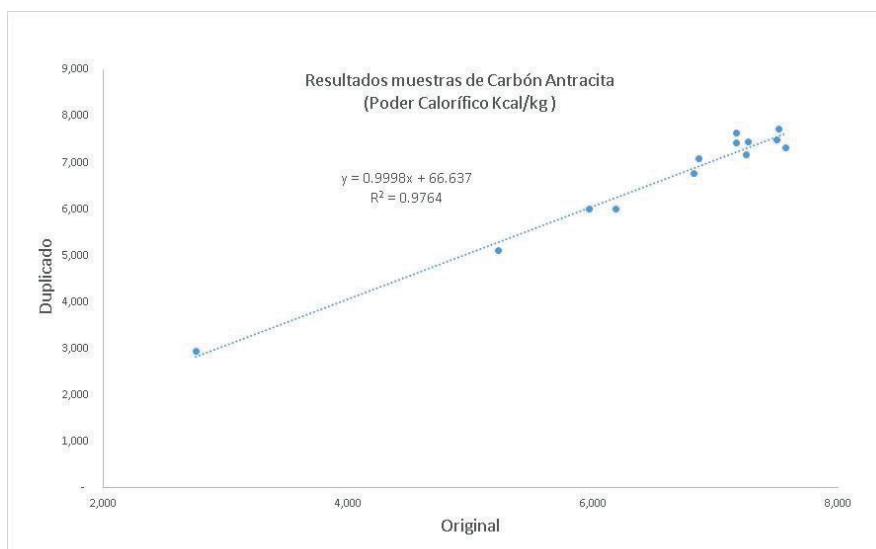

Figura 7. Resultados muestra original y duplicado (poder calorífico -Kcal/kg)

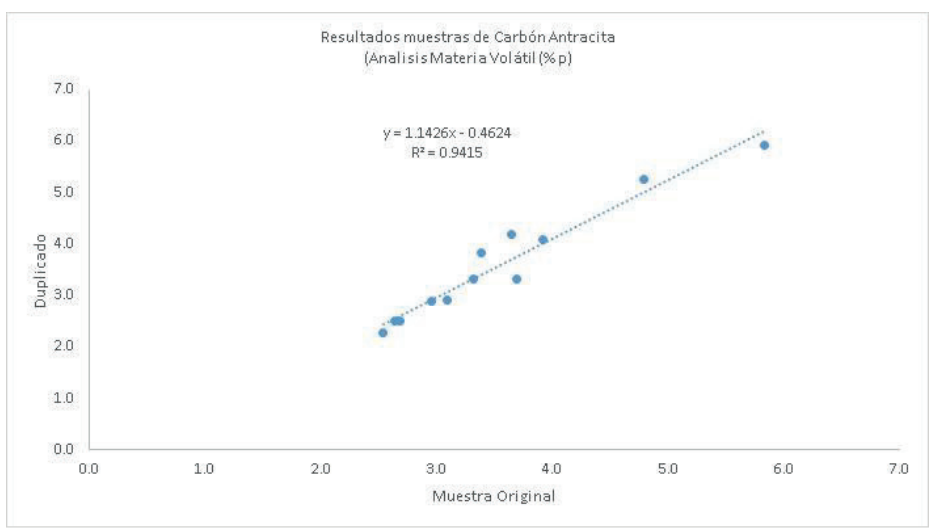

Figura 8. Resultados muestra original y duplicado (contenido de material volátil - \%)

En la Figura 9 se presenta el histograma de carbón fijo en base seca de los resultados de las muestras recolectadas en geología de campo y perforaciones diamantinas.

En la Figura 10 se presenta el histograma del poder calorífico en base seca de los resultados de las muestras recolectadas en geología de campo y perforaciones diamantinas.

En la Figura 11 se muestra el scatter plot del contenido de carbón fijo y contenido de cenizas para los datos recolectados en campo y perforaciones diamantina.

\subsection{Interpretación y modelo geológicos}

Basado en los resultados obtenidos de geología de campo y perforaciones diamantina se realizó la interpretación geológica en el depósito carbonífero.

Se realizó la estimación de recursos minerales siguiendo los criterios para la estimación de Recursos de carbón según lo indicado en el documento Paper 88-21 de acuerdo con Geological Survey of Canada (Hudges, J.D., Klatzel-Murdy, L., Nikols, 1989) de NI-43101 para los depósitos de carbón antracita. En la Tabla 5 se presentan los 


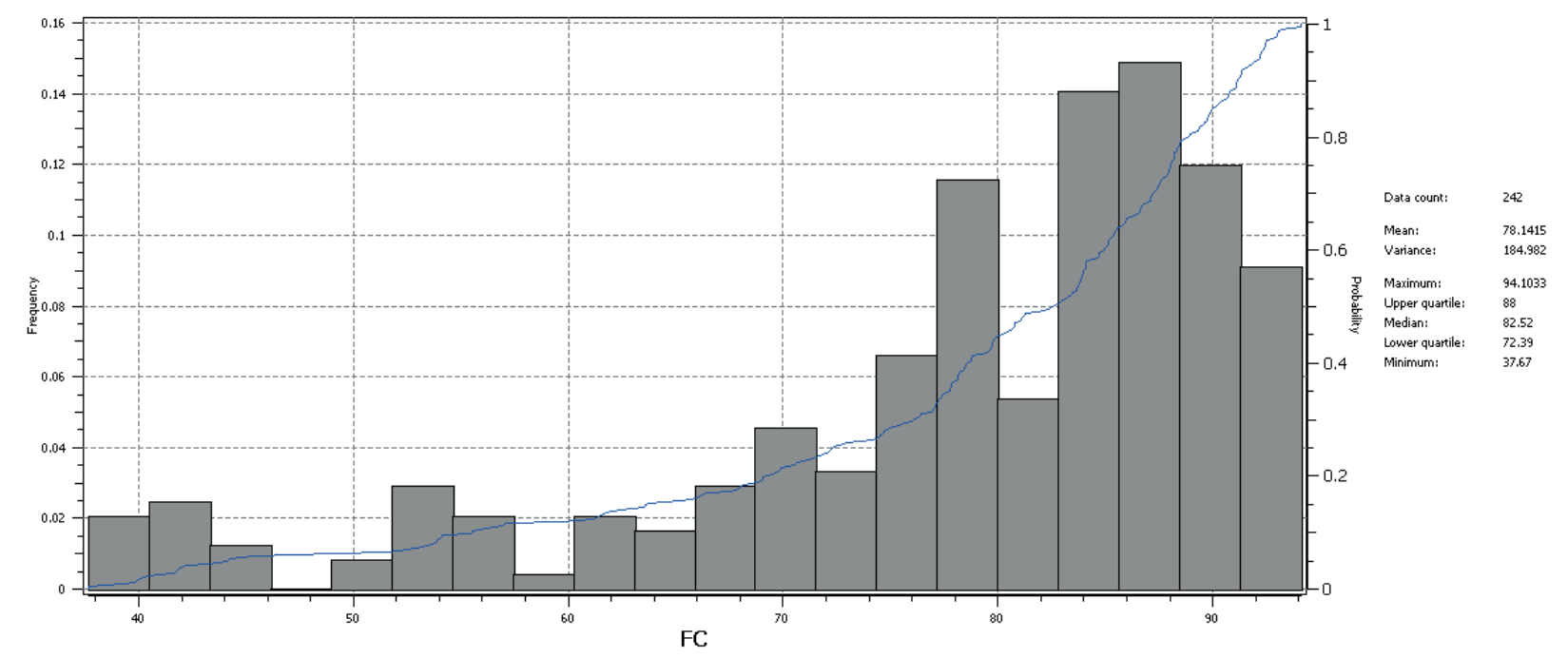

Figura 9. Histograma de carbón fijo expresado en \%

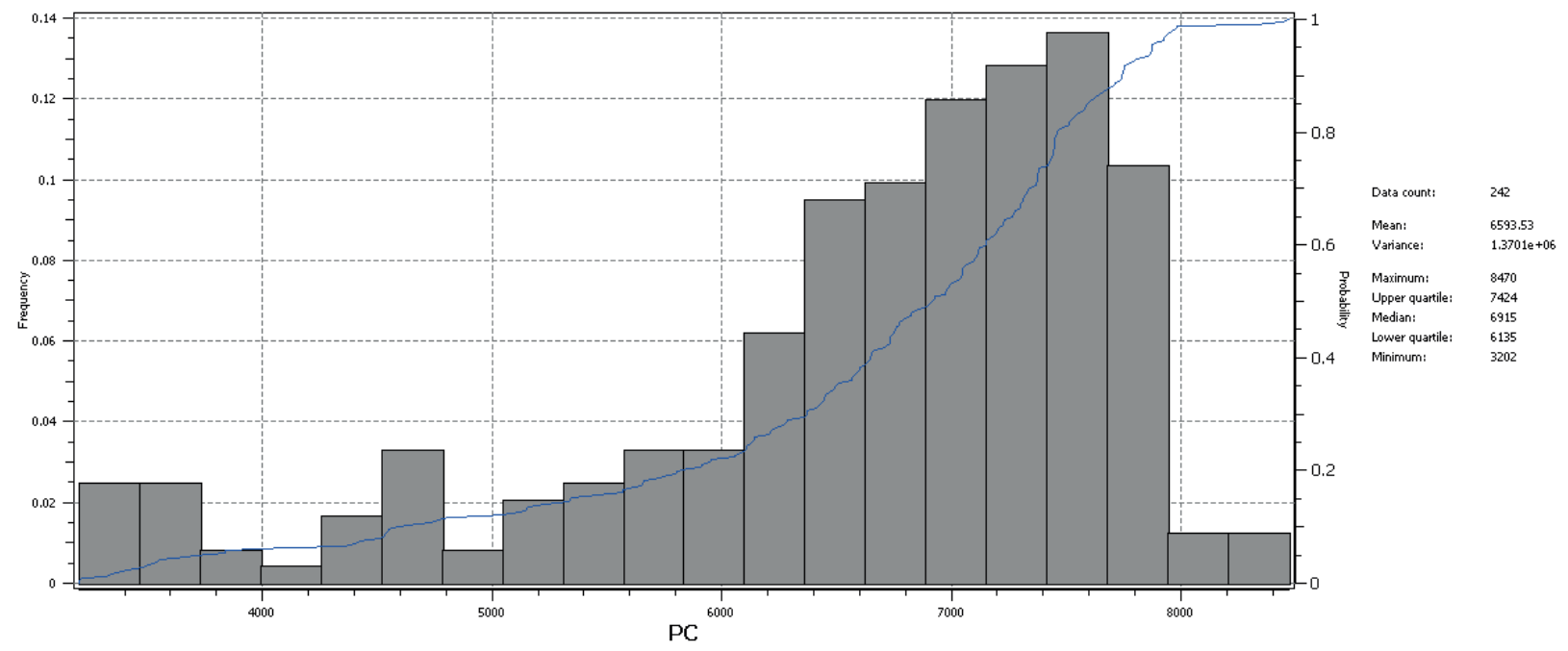

Figura 10. Histograma de poder calorífico expresado en $\mathrm{Kcal} / \mathrm{kg}$

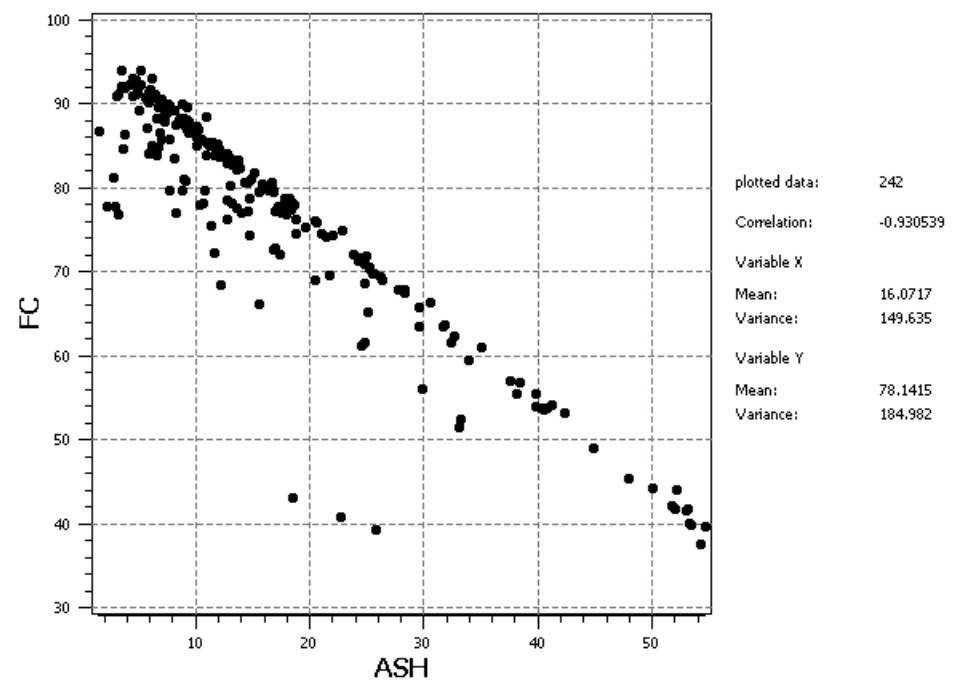

Figura 11. scatter plot - cenizas vs carbón fijo 
criterios establecidos en el paper 88-21 para la estimación de Recursos Minerales de carbón.

Aplicando los criterios de norma antes mencionada, se clasificó el depósito con una geología de tipo complejo dado que geotécnicamente la zona de estudio ha estado sujeta a extrema deformación tectónica, asimismo, se puede verificar la secuencia estratigráfica normal. Por otro lado, se clasificó el depósito como tipo subterráneo $\mathrm{y}$ en consecuencia los métodos de minado deberían ser convencionales tales como shirinkage y room and pillar.

Los parámetros de cuantificación como número de puntos de muestreo, espesor, área, densidad fueron determinados de las perforaciones diamantina. Basados en esta información se estimó y clasificó la cantidad de recursos de carbón antracita para la zona de estudio, en las categorías de acuerdo con su factibilidad de extracción como medidos, indicados e inferidos, no se consideró recursos especulativos en el estudio, pero pueden representar un potencial, dada las características del depósito. Para la categoría de recursos medidos se consideró un espaciamiento promedio de 100 metros, para recursos Indicados una distancia promedio de 200 metros y los recursos inferidos 400 metros con 3 puntos de referencia en todos los casos.

\section{DISCUSIÓN}

Las principales características comerciales del carbón antracita son el poder calorífico, carbón fijo, material volátil, contenido de cenizas, contenido de azufre y humedad. Durante las actividades de geología de campo y actividades de exploración mediante perforación diamantina, se identificó características de las muestras de carbón para ser enviadas a los laboratorios.

Las actividades de campo fueron el primer paso para conocer el depósito minero. Se desarrolló la evaluación geológica, identificación de mantos de carbón, toma de muestras, análisis de laboratorio, validación de resultados, interpretación geológica, desarrollo de modelo espacial geológico.

Se utilizó herramientas para la definición de los lugares potenciales para la perforación diamantina. En ese sentido, se seleccionó una concesión minera las cuales se continuaría los estudios geológicos tales como la perforación diamantina y estudios geotécnico e hidrogeológicos.
El programa de calidad se aplicó a las actividades de exploración y permitió obtener información válida y confiable para la interpretación geológica y estimación de Recursos basados en norma NI 43101 - paper 88-21 (Hudges, J.D., Klatzel-Murdy, L., Nikols, 1989).

Con los resultados de laboratorio de las muestras de geología de campo y análisis estadístico se elaboró las zonas potenciales de exploración y se incluyó el análisis de las variables ambientales, sociales, económicas del proyecto fueron la base para la ubicación de los lugares a perforar.

Con los resultados obtenidos durante la perforación se incrementó la base de datos del proyecto, y con la aplicación de técnicas estadísticas aplicadas a los datos obtenidos en los trabajos de exploración se logró contar con una base confiable para las estimaciones de Recursos asociadas al proyecto minero.

Tal como se presenta en la Figura 7 y Figura 8, los análisis de muestras original y duplicado asociadas al poder calorífico y material volátil, han mostrado correlaciones estadísticas buenos con $\mathrm{R}^{2}$ igual 0.97 y 0.94 respectivamente.

Los resultados de laboratorio muestran correlaciones importantes entre las características más relevantes del carbón antracita en el área del proyecto. Por ejemplo, de la Figura 9 muestra que más del $70 \%$ de las muestras analizadas presentan contenidos de carbón fijo superiores a $87 \%$ en base seca. En el caso del poder calorífico, el 70\% de las muestras analizadas presentan valores de 7,000 Kcal/ $\mathrm{Kg}$ en base seca. Asimismo, existe una relación coherente entre el contenido de carbón fijo y contenido de cenizas, esto se muestra en la Figura 11 que para contenidos carbón fijo $88 \%$ equivale valores de $10 \%$ de cenizas.

La información geológica obtenida de los trabajos de exploración de geológica de campo y perforación diamantina, muestran resultados coherentes y estadísticamente confiables para ser utilizados en las siguientes etapas del proyecto.

El programa de calidad incluye el aseguramiento de la calidad y control de calidad de los datos geológicos que permiten obtener la estimación de recursos y posteriormente incorporar los factores para la estimación de reservas.

Tabla 5. Criterios de clasificación de Recursos de Carbón - Paper 88-21 (CSA, 2011)

\begin{tabular}{|c|c|c|c|c|}
\hline \multirow{2}{*}{ Tipo de Geología } & \multirow{2}{*}{ Criterio } & \multicolumn{3}{|c|}{ Aseguramiento de la categoría existente } \\
\hline & & Medidos & Indicados & Inferidos \\
\hline \multirow{4}{*}{ Complejo } & Espacio en sección Trasversal (m) & 150 & 300 & 600 \\
\hline & Número mínimo de datos por sección & 3 & 3 & 3 \\
\hline & Espacio medio entre puntos de datos a lo largo de la sección (m) & 100 & 200 & 400 \\
\hline & Espacio máximo entre puntos de datos a lo largo de la sección (m) & 200 & 400 & 800 \\
\hline
\end{tabular}




\section{CONCLUSIONES}

El Programa de Calidad (PDC) es una herramienta de gestión en la actividad minera y en las actividades de exploración de los proyectos mineros cual sea su naturaleza.

- $\quad$ Es parte del Programa de Gestión del Proyecto.

- El Programa de Calidad es aplicado a todas las etapas del proyecto minero de exploración tales como inicio, planificación, ejecución, control y monitoreo y cierre.

- Permite tener estándares de calidad alineados con las mejores prácticas de la industria minera y aceptadas internacionalmente tales como la Norma NI-43101 y en el caso de industria de Carbón el paper 88-21.

- Permite asegurar la calidad de los resultados obtenidos durante el desarrollo del proyecto de manera temprana y así obtener información válida para la elaboración de estudios de estimación de recursos y reservas del proyecto

- Permite el control de lo calidad y corrige las desviaciones u errores.

- Da confianza a los inversionistas, bancos de inversión, dueños de las empresas sobre el valor de los activos mineros.

- Permite proyectar y programar de manera confiable las etapas posteriores de los proyectos mineros.

\section{AGRADECIMIENTOS}

Los autores del presente artículo quieren extender sus agradecimientos a la Unidad de Posgrado de la Universidad Nacional Mayor de San Marcos por su apoyo académico.

\section{REFERENCIAS}

Abbott, D. M. (2007). Assuring the Reliability of Your Sampling Results. AIPG, December, 33-38. https://www. ammoniteresources.com/pdf/Assuring_the_Reliability.pdf

Carrascal Miranda, R., Matos Ávalos, C., \& Silva Campos, O. (2000). Carbón en el Perú. INGEMMET. Boletín, Serie B: Geología Económica; $N^{\circ}$ 7, 150. https://repositorio. ingemmet.gob.pe/handle/20.500.12544/234

CIM - Canadian Institute of Mining, M. and P. (2018). Mineral Exploration Best Practices Guidelines. https:// mrmr.cim.org/media/1130/cim-mineral-exploration-bpguidelines_2018.pdf

CSA. (2011). NATIONAL INSTRUMENT 43-101 STANDARDS OF DISCLOSURE FOR MINERAL PROJECTS. 2011, 7043-7086. https://mrmr.cim.org/media/1017/nationalinstrument-43-101.pdf

Dunin-Borkowsk, E., Jacay, J., \& Sánchez-Izquierdo, J. (2007). Génesis del carbón peruano en el marco de la tectónica global. Revista Del Instituto de Investigación de La Facultad de Ingeniería Geológica, Minera, Metalurgica y Geográfica, 10(19), 7-27. https://revistasinvestigacion. unmsm.edu.pe/index.php/iigeo/article/view/379/323

Hudges, J.D., Klatzel-Murdy, L., Nikols, D. J. (1989). A standardized coal resource/reserve reporting system for Canada. In Geological Survey of Canada GSC Paper 88-21 (Vol. 88, Issue 21, p. 46). https://ftp.maps.canada. $\mathrm{ca} / \mathrm{pub} /$ nrcan_rncan/publications/STPublications PublicationsST/126/126809/pa_88_21.pdf

ISO. (2015). Quality management systems - Fundamentals and vocabulary. In English (Vol. ISO9000). https://www.iso.org/ obp/ui/es/\#iso:std:iso:9000:ed-4:v1:en

PMI. (2017). PMBOK Guide | Project Management Institute. In $P M B O K$ Guide. https://www.pmi.org/pmbok-guidestandards/foundational/PMBOK 